\section{Preventive and Social Pediatrics}

\section{GENDER AND HEALTH RISK BEHAVIORS IN TRAFFIC AMONG HIGH SCHOOL STUDENTS IN ZAGREB}

Natko Gereš*, Iva Lukačević-Lovrenčić, Aida Mujkić. Psychiatric hospital Sveti Ivan Zagreb

\subsection{6/archdischild-2021-europaediatrics.457}

Risky traffic behaviors among youth potentially lead to serious consequences, injuries or deaths. Young men are particularly at risk.

Growing body of research indicates that the endorsement of traditional masculinity is at the root of the causes of deviant behavior of boys. With National program for road safety due to expire in 2020, there is a need for evidence on the present state of behaviors of youth in traffic in Croatia, using indicators that take into the account the differences between boys and girls and the influence of the prevalent gender norms on these groups.

The goal of the research was to analyze the health risk behaviors in traffic for high school students from Zagreb by sex as well as to test the association between the endorsement of traditional masculinity and risky traffic behaviors among adolescent boys.

Study and included 4094 high school students in the City of Zagreb. Two of the most prevalent risky traffic behaviors were analyzed: the use of seat belts while riding in the front seat of a vehicle and the use of helmets while riding a bike. To compare scores between participants who reported risky traffic behavior and those who did not, we used chi-square test for sex and independent samples t-test for masculinity scale.

Students engage in multiple health risk behaviors while participating in the traffic. Over half of the students report inconsistent seat belt use when riding in the front seat of the car and only $7.5 \%$ report ever using a helmet when riding a bike. A significant association was found between sex and inconsistent seat belt use, with male adolescents reporting significantly more risky behavior than their female counterparts. Stronger endorsement of traditional masculinity norms among boys was correlated with less consistent use of seat belts $(p<0.001)$. The difference between male and female students in carrying a helmet when riding a bike was less significant, with results suggesting that the lack of protection when riding may be a part of the general youth culture.

The results in general highlight the prevalent harmful risky traffic behaviors of adolescents. The study highlights the need to further investigate the causes of risky traffic behaviors among youth and intervene at the level of youth culture. The results further suggest the need to design experimental gendertransformative interventions for boys specifically, as a group at a higher risk.

\section{PREVALENCE OF SMOKING AND ALCOHOL CONSUMPTION AMONG RUSSIAN ADOLESCENTS}

Julia Levina*, Leila Namazova-Baranova, Kamilla Efendieva, Anna Alekseeva, Elena Vishneva, Valery Zvonarev. The Research Institute of Pediatrics and Child Health of the Central Clinical Hospital of the Russian Academy of Sciences of the Ministry of Science and Higher Education of the Russian Federation

10.1136/archdischild-2021-europaediatrics.458
Objective Smoking is one of the main preventable causes of death, but nonetheless leads to more than 7 million deaths in the world each year. Worldwide, 3 million deaths every year result from harmful use of alcohol. Russia is facing a serious burden of smoking and alcohol consumption, which affects not only adults, but also adolescents. In order to conduct appropriate tobacco and alcohol control policies, it is important to understand smoking and alcohol consumption prevalence.

Methods An analysis of the prevalence of smoking and alcohol consumption was carried out in the research center in Moscow, Russian Federation among 303 adolescents (84 boys and 219 girls) 15-18 years old who participated in a study of the prevalence of allergic diseases according to the protocol of the research program GA2LEN. All participants completed the standardized GA2LEN questionnaire.

Results $25 \%$ of respondents (11\% of boys and $14 \%$ of girls) said they had smoked for as long as a year, among them, most started smoking at the age of $16.68 \%$ of teens who said they were still smoking as of one month ago, smoked 10 cigarettes a day. Among participants who had previously smoked, $55.1 \%$ already stopped smoking or started to smoke less, most of them at the age of 18. Among those who stopped smoking, they had smoked 12 cigarettes per day in average. $27 \%$ of participants were regularly exposed to tobacco smoke in the last 12 months. $34.2 \%$ of respondent's fathers smoked regularly during their childhood, $7.9 \%$ of respondents had mothers who smoked regularly during their childhood/before they were born. 3.9\% of respondents said that they had been hospitalized before the age of two years for lung disease We found a statistically significant association between a mother smoking during childhood or before the birth of a child and that child being hospitalized before the age of two years for lung disease $(p=0,001)$.

$53 \%$ of respondents $(14 \%$ of boys $39 \%$ of girls) said they never drank any alcohol. $31 \%$ of participants $(7 \%$ of boys and $24 \%$ of girls) said they drank alcohol less than once a week, $9 \%$ of respondents (5\% of boys and $4 \%$ of girls) said they drank alcohol at least once a week but less than once a day.

Conclusion Further efforts should be devoted to encouraging smoking and alcohol cessation and to develop strategies for preventing tobacco and alcohol use in young patients.

\section{\begin{tabular}{|l|l}
459 & THE IMPACT OF TOXIC STRESS ON A DEVELOPING
\end{tabular} BRAIN}

Vanja Slijepcevic Saftic*, Gordana Buljan Flander, Ella Selak Bagarić. Zagreb Child and Youth Protection Center

\subsection{6/archdischild-2021-europaediatrics.459}

The effects of negative environmental factors in childhood result in neuro-biological changes. Exposed to powerful, negative environment, lead to hyperactivation of the neurological and endocrine system. This state is known as a toxic stress. Symptoms may frequently imitate ADHD and other neurodevelopmental disorders. Hyper-vigilance and dissociation, could be mistaken for inattention. Impulsivity might be brought on by a stress response in overdrive.

The aim of this study was to estimate preliminary results of a multi-disciplinary (Pediatric neurologist, Social worker, Psychologist, Psychiatrist, Speech therapist and special need 
expert) clinical assessment of patient came to Child and Youth protection Center, Zagreb, Croatia, in case of adverse childhood experiences, acording ACEs (Adverse Childhood Experience score) $\geq 4$. From 2015 - 2019 in Child and Youth Protection Center Zagreb we prospectively observed 1053 children with $\geq 4$. We were looking for presence of minor neurological dysfunctions (gross motor function, mild dysfunction in posture, reflexes, coordination, fine manipulative ability, fine motor disfunction, dyskinesia and excessive associated movements). For those with ACES $\geq 4$, and neurodevelopmental delay, EEG was indicated. We also evaluated simptoms of impulsivity, hyperactivity, and an inability to focus.

Neglect, physical and emotional abuse and high conflict divorce are the most important adverse experiences resulting in deviations in neurodevelopment.

Impulsive behaviour, and other form of ADHD 'like' variants are significantly more frequent (over 50\%) in children with history of some kind of abuse or neglect. Over $70 \%$ of patients with ACES $\geq 4$ showed dysrhytmic or other form of nonspecific changes in EEG.

In order to understand better the processes of the effects of toxic stress on the developing brain and identify forms of intervention, we have to apply several levels of study - scientific and theoretical, empirical and professional. An integrative paradigm in an approach to neurodevelopmental disorders caused by negative environmental factors provides the conceptual framework for further insight.

\section{THE INTEGRATION OF CHILDREN AND ADOLESCENTS WITH DEVELOPMENTAL DISABILITIES}

Željka Karin* ${ }^{*}$ Tonka Karin. Public Health Institute of Split and Dalmatian County

10.1136/archdischild-2021-europaediatrics.460

Introduction In Croatia, any child having developmental disabilities with an essential need of school educational support is entitled to accessibility and inclusion in order to be provided with free primary and high school education. A psychosomatic disorder in child is determined by the specialist of school and adolescent medicine who along with the team of school expert associates (psychologist, pedagogue, defectologist) is responsible for ensuring an adequate form of school education and support for the eligible child.

Within his activities at the primary level of healthcare in the public health system, the school doctor continuously monitors health condition and abilities in children and adolescents, the implementation of therapeutic, rehabilitation and other professional practices as well as the school education adjustment meeting the needs of persons with health disabilities.

The Aim To present the results of the psychosomatic assessment in children as well as to determine the adequate programme of the elementary and high school education for school children with developmental disabilities. To report on most common disabilities affecting the educational attainment and functioning in school children.

The subjects and methods: By using retrospective data analysis of the annual reports comprising 172 school medicine teams in the Republic of Croatia from the elementary and high school children, the proportion of children who due to developmental disabilities were provided with an adequate school education programme, was determined. In children with disabilities an adequate form of school education was determined by gender and school, as well as most common disabilities affecting educational attainments.

The results: When establishing psychosomatic condition in children due to developmental disabilities for 5.2\% of elementary school children and $1 \%$ of high school children, one of the school education forms was determined. In relation to school children provided with the adequate form of school education in elementary school 37\% girls followed regular programme with an individual approach, 49\% girls were provided with an individual approach and adjusted programme, $6 \%$ girls with a special programme, whereas $8 \%$ girls were in the programme for acquiring competences. Within regular programme an individual approach in school education was provided for $45 \%$ elementary school boys, an individual approach and adjusted programme for $41 \%$ boys, a special programme for $5 \%$ boys, and in the programme for acquiring competences there were $9 \%$ boys. In high school within the regular programme, an individual approach was provided for $41 \%$ females and 39\% males, an individual approach and adjusted programme were followed by $21 \%$ females and $20 \%$ males, special programme was provided for $11 \%$ females and $13 \%$ males, whereas in the programme for acquiring competences and individual approach were $27 \%$ females and $28 \%$ males.

In children provided with an adequate form of school education most common diagnoses according to DSM-10 classification affecting school attainments and functioning of children related to voice and speech disorder as well as specific learning difficulties in $37.9 \%$, central nervous system impairment in $20.7 \%$, mental disorder and behaviour disorder in $19.5 \%$, and intellectual disabilities in $14.2 \%$ children.

The conclusion: The school child with developmental disabilities requires educational support and education mandatory for all children having developmental disabilities in order to enable positive health-related and educational outcomes along with maintaining and promoting physical and mental health. In order to facilitate school education of children with developmental disabilities and to promote developmental potentials in children, a multidisciplinary approach is essential to make disability assessment in children, to estimate their abilities and capacities as well as to determine developmentally appropriate education and support.

\section{HOW CAREGIVER'S HOSPITAL CONSULTATION TIMING IS INFLUENCED BY THE CARE TARGET II -IN CASE OF ASD, ADHD, ALLERGIC OR OTHER DISEASES}

Juri Ogawa*, H Tajima*, M Kakinuma*, M Takarabe*, Y Momota, I Nose, M Takahashi. Nippon Medical School

\subsection{6/archdischild-2021-europaediatrics.461}

The timing of consulting the hospital is important to ensure the quality of life of the child. Avoiding the delay in consulting is very important as well avoiding unnecessary visit. Based on the study with veterinary study on visitation timing, we speculate that caregivers may not be making the judgement purely on child's physical condition, but basing on the expression of the pain or difficulties of taking the child to the hospital. Therefore, it is important to understand how disease, disabilities specific behavior of the child influences the hospital consultation timings or frequencies by the caregiver. If the consultation timings are influenced by child's behavior, or characteristics, preventive education would be possible for the 